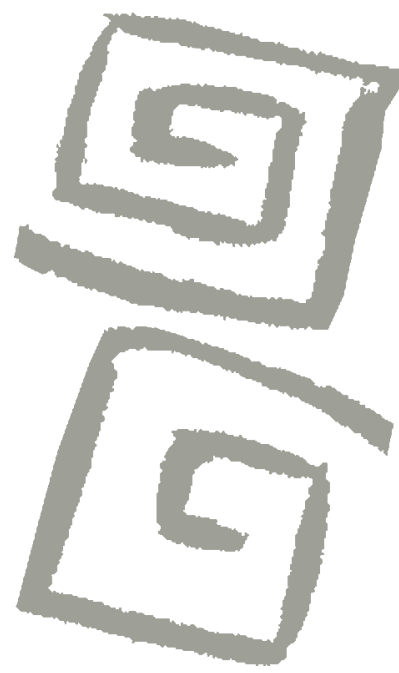

\title{
Significados en torno al desarrollo del consumo problemático y la dependencia alcohólica en comunidades mapuches rurales de la región de la Araucanía, Chile, 2016-2017
}

\author{
Meanings regarding the development of problematic \\ consumption and alcohol dependence in rural Mapuche \\ communities of the Araucanía region, Chile, 2016-2017
}

\author{
Gabriela Orieta Garcés Pérez ${ }^{1}$, Alba Ximena Zambrano Constanzo ${ }^{2}$
}

'Antropóloga, Magíster en Salud Pública Comunitaria y Desarrollo Local. Docente, Universidad Católica de Temuco, Temuco, Chile. $\square$ (iD

${ }^{2}$ Psicóloga, Doctora en Psicología Social. Directora, Departamento de Psicología, Universidad de la Frontera, Temuco, Chile. $\bowtie$ (ID)
RESUMEN Esta investigación indaga las concepciones, significados y prácticas involucradas en el desarrollo del consumo problemático y la dependencia alcohólica en comunidades mapuches rurales del sur de Chile, en los años 2016 y 2017. Se caracterizan significados en torno al consumo de riesgo, escenarios y procesos protectores de la salud y de riesgo, e identifican elementos socioculturales para la proyección de actuaciones preventivas. La metodología empleada fue de tipo cualitativa-etnográfica. Se realizaron nueve entrevistas en profundidad a personas adultas con consumo de riesgo, recuperadas y no consumidoras de alcohol, y observaciones etnográficas en talleres participativos con la comunidad. Los resultados vinculan procesos de transculturación, pérdida y vitalidad cultural a dinámicas sociorelacionales que están en la base del desarrollo del consumo problemático de alcohol, lo que configura un escenario de alta complejidad que requiere, en su abordaje, la superación de la dicotomía factores de riesgo/protección.

PALABRAS CLAVES Alcoholismo; Prevención Primaria; Población Indígena; Aculturación; Chile.

ABSTRACT This research looks in to the conceptions, meanings and practices involved in the development of problematic consumption and alcohol dependence in rural Mapuche communities in southern Chile in the years 2016-2017. It characterizes the meanings surrounding at-risk consumption as well as scenarios and processes that protect health and those that facilitate risk, and identifies sociocultural elements to potentiate preventive actions. The methodology used was qualitative-ethnographic. Nine in-depth interviews were conducted with adults with at-risk consumption, recovered alcohol users, and nonconsumers of alcohol, and ethnographic observations were carried out in participatory workshops with the community. The results link processes of transculturation, loss and cultural vitality with socio-relational dynamics that are at the base of the development of problematic alcohol consumption, marking a scenario of high complexity that requires overcoming the traditional dichotomy of risk/protection factors.

KEY WORDS Alcoholism; Primary Prevention; Indigenous Population (Public Health); Acculturation; Chile. 


\section{INTRODUCCIÓN Y ANTECEDENTES TEÓRICOS}

La Organización Panamericana de la Salud $(\mathrm{OPS})^{(1)}$ señala que el alcohol es una de las sustancias más usadas y causantes del mayor daño entre las comunidades indígenas en la Región de las Américas. Esta población es especialmente vulnerable dados sus niveles de pobreza y el limitado acceso a la salud, la educación y otros servicios apropiados para la prevención y tratamiento de los problemas relacionados con el consumo de alcohol, influyendo también los complejos procesos de colonización por ellos vividos. Las consecuencias más significativas del consumo de alcohol en las comunidades indígenas están asociadas con problemas de la salud, el daño social, y la destrucción de la cultura y los valores indígenas. Sin embargo, es escasa la información producida sobre cómo abordar y reducir los problemas sociales y de salud relacionados con el alcohol entre estas comunidades; acerca de cuáles son los recursos existentes en las comunidades para enfrentar este problema y qué políticas funcionarían de manera específica con esta población vulnerable ${ }^{(1,2,3)}$.

En Chile, el alcoholismo constituye el primer factor de riesgo que causa más muerte y discapacidad en el país, según el "Estudio de carga de enfermedad y carga atribuible $^{\prime \prime(4)}$, el consumo de alcohol se relaciona con el $12,4 \%$ de los años de vida saludables (AVISA) perdidos por muerte o discapacidad. Chile se sitúa en el primer lugar en cantidad de consumo de alcohol per cápita al año en Latinoamérica, con un total de 9,6 litros de alcohol puro per cápita en adultos ${ }^{(5)}$.

En la región de la Araucanía, el diagnóstico de determinantes sociales de la salud ${ }^{(6)}$ y la última Encuesta de consumo de alcohol en población general(7), indican que la región presenta un consumo por sobre el promedio nacional y el nivel más alto de bebedores problema del país, que llega a un 21,6\%, aumentando a $36,5 \%$ en el caso de los hombres. Esta situación se agudiza debido al alto porcentaje de ruralidad, donde tiende a existir una actitud más permisiva con respecto al consumo de alcohol que en el ámbito urbano, en especial, en el rango etario de 45 a 64 años $^{(6)}$.

En la Araucanía, la población perteneciente a pueblos originarios asciende a un $32,0 \%$, la mayor proporción a nivel nacional ${ }^{(8)}$. Respecto a los indicadores de desarrollo, la Araucanía presenta el índice de desarrollo regional más bajo a nivel país $(0,413)$, el que sintetiza seis dimensiones: educación (lugar 10/15), salud (12/15), conectividad (15/15), bienestar socioeconómico (14/15), actividad socioeconómica $(14 / 15)$ y seguridad $(7 / 15)^{(9)}$.

Desde una perspectiva histórica, el consumo de alcohol en esta región se relaciona con las dinámicas de conquista y colonización del territorio, en las que el alcohol fue utilizado como instrumento para la ocupación y apropiación de ese territorio $^{(10)}$. Posteriormente, con la anexión del territorio del sur del río Biobío al Estadonación, y la conformación del campesinado chileno-mapuche, el alcohol fue utilizado como medio de pago, y en un contexto de inestabilidad económica e inflación, la estabilidad de su valor le convirtió en medida estándar de intercambio comercial y fue incorporado en la canasta básica de alimentos $^{(11)}$. En las zonas rurales de la región, se observa un consumo de alcohol arraigado en la identidad campesina mapuche y no mapuche, vinculado a las faenas agropecuarias y a formas de reciprocidad y ayuda mutua en el trabajo, (como el mingako, colaboración comunitaria en faenas productivas; el Rukán, construcción de vivienda) y a formas de sociabilidad comunitaria ${ }^{(12)}$. Estas dinámicas históricas y patrones de consumo problemático están presentes en las comunidades mapuche del sector costero de la región de la Araucanía, en las que existe una alta prevalencia de consumo de alcohol y una baja percepción del riesgo declarada por los equipos de salud locales ${ }^{(13)}$.

La colonización modificó los patrones tradicionales de consumo, cambiando un uso ocasional y limitado a ciertas festividades, a un uso profano indiscriminado, debilitando las normas que restringen el uso y la 
autoridad de los líderes indígenas en su regulación ${ }^{(14,15)}$. Las consecuencias del consumo se relacionan con el deterioro en la salud y la calidad de vida de las personas $y$, a nivel sociocomunitario, con distintos tipos de violencia familiar y comunitaria. La dificultad de la medición del consumo de alcohol en pueblos originarios y sus implicancias, conforma otra arista del problema ${ }^{(1)}$. Habría un subregistro tanto en lo reportado en las estadísticas sanitarias ${ }^{(16)}$, como en las encuestas de consumo individual, que muestran un sesgo hacia la baja y explican, en parte, la brecha observada entre las estadísticas de ventas y las de encuestas de consumo individual ${ }^{(17)}$.

Según señalan Menéndez y Di Pardo ${ }^{(18)}$, el consumo problemático de alcohol abarca un conjunto de relaciones complejas y en constante cambio, cuyo abordaje requiere enfrentar metodológicamente el problema en lugar de simplificar la realidad, describiéndola a través de variables y de categorías aisladas. Es así como, en esta investigación, entenderemos por consumo problemático de alcohol, a aquel cuya frecuencia e intensidad tiene impacto directo e indirecto en distintas áreas de la vida de la persona, produciendo consecuencias negativas tanto para quien la consume, como para terceras personas (contexto familiar, laboral, seguridad pública) ${ }^{(19)}$.

En esta perspectiva, la investigación toma como marco de referencia el enfoque teórico metodológico de la epidemiología sociocultural, aproximación dialógica al fenómeno de la enfermedad como proceso colectivo, que se nutre de saberes y recursos reconocidos y no reconocidos como científicos ${ }^{(20,21)}$. Desde este enfoque, la salud constituye una expresión eminentemente relacional, que sintetiza diversas esferas de la calidad de vida ${ }^{(20)}$, por tanto, de igual forma para su comprensión y abordaje, se debe incluir no solo los diferentes factores que operan respecto del problema determinado, sino al conjunto de actores sociales significativos que viven, sufren y actúan respecto de dicho problema, es decir, se plantea la necesidad de incluir en la investigación de los procesos de salud/ enfermedad/atención, aspectos socioculturales y económico-políticos, junto con los biológicos y ecológicos ${ }^{(21)}$. De esta forma, se busca comprender la forma en que la salud y la enfermedad encajan en todo el modo de vida, considerando que los miembros de una comunidad, por lo general, comparten actitudes sobre lo que significa ser saludable, y qué tipos de comportamientos hacen a una persona "sana"(22).

Asimismo, en este estudio se asume desde una perspectiva crítica, que los procesos de salud/enfermedad/atención, se sitúan en lógicas de poder político-económico propias del sistema social que los engloba ${ }^{(23,24,25)}$. Desde esta arista se señala que la aceptación del alcoholismo como una enfermedad del cuerpo, mente o familia, despolitiza eficazmente el problema, normalizando las condiciones socioeconómicas que lo producen. Esto último se expresa en que las acciones político-institucionales buscan adecuar las percepciones y comportamientos de los sujetos y colectivos al orden social $^{(23)}$, seleccionando y definiendo lo protector o lo riesgoso en función de los fines del sistema hegemónico.

En consecuencia, nos adherimos al planteamiento de la construcción social y cultural del riesgo. Desde esta perspectiva, la percepción pública del riesgo y los niveles de aceptación del mismo son construcciones colectivas. En palabras de Douglas y Widalvski(26) "cada forma de organización social está dispuesta a aceptar o evitar determinados riesgos". Además, desde esta misma perspectiva en las culturas originarias, se advierte la presencia de procesos protectores de la salud como construcciones sociales, que se tornan beneficiosos para las personas, familias y comunidades, se convierten en un favorecedor de las defensas y soportes, y estimulan una direccionalidad favorable a la vida humana, individual y/o colectiva ${ }^{(25)}$. Por tanto, también interesa comprender e identificar estos escenarios y procesos protectores de la salud que forman parte de sus mundos de la vida.

Cabe destacar que, desde perspectivas de análisis crítico, la noción de trauma coIonial $^{(27,28,29)}$ contribuye a la comprensión del consumo problemático de alcohol en población indígena. Desde esta aproximación se 
historiza el problema del consumo en las comunidades originarias y visibiliza la dimensión colectiva del trauma reproducido transgeneracionalmente. Se plantea que es una forma única de trauma, por su carácter complejo, continuo, colectivo y acumulativo ${ }^{(27)}$, y estaría vinculado directamente con la prohibición de prácticas culturales, y con políticas e instituciones de asimilación. Se señala que el trauma histórico afectaría a la mente, las emociones y el espíritu de las poblaciones indígenas ${ }^{(28)}$ y estaría vinculado a la mayor prevalencia de problemas de salud mental en esta población. La información disponible sobre la salud mental de los pueblos indígenas indica que estos experimentan altas tasas de depresión, alcoholismo, suicidio y violencia, problemas que se relacionan con la discontinuidad cultural y la opresión a la cual están sujetos ${ }^{(30)}$.

La investigación producida sobre el consumo de alcohol en pueblos originarios, ha relacionado los patrones de consumo problemático con procesos de transculturación, pérdida y revitalización cultural de estos pueblos $^{(31)}$. Estudios de tipo correlacional realizados en México y Costa Rica señalan una relación estadísticamente significativa entre la autoestima y la valoración positiva de la identidad étnica. El primer estudio ${ }^{(32)}$ constata que el compromiso con las prácticas étnicas en jóvenes que provenían de "reservas indígenas", estaba asociado con sentimientos de alta autoestima, y el segundo estudio(33) concluye que una identidad étnica positiva, ligada a la autoestima personal, serviría para combatir la discriminación, correlacionando negativamente con medidas de aislamiento y depresión ${ }^{(32)}$. Por otra parte, investigaciones realizadas en EEUU con pueblos originarios señalan que la identidad cultural tiene un rol protector en el desarrollo de síntomas depresivos en jóvenes indígenas y que una mayor participación en actividades tradicionales y mayor apropiación del lenguaje tribal se relacionaron con un menor riesgo de síntomas depresivos teniendo en cuenta otros factores individuales y familiares asociados a la presencia de estos síntomas. A su vez, los testimonios de ancianos nativos americanos señalan que muchos de los problemas de abuso de sustancias están relacionados con la pérdida de la cultura tradicional ${ }^{(34)}$.

De lo anteriormente expuesto podemos derivar que la evidencia producida sustenta la incidencia de los significados atribuidos a la identidad cultural y las formas de vivenciar y valorar la cultura, con el desarrollo de problemas de salud mental y consumo de alcohol, en las trayectorias de vida de personas y colectividades. Por tanto, la investigación realizada tuvo como propósito comprender concepciones, significados y prácticas socioculturales involucradas en el desarrollo del consumo problemático y dependencia alcohólica en personas mapuche de comunidades rurales del sector costero de la región de la Araucanía, a partir de la identificación de concepciones en torno a lo problemático del consumo, la caracterización de escenarios y procesos protectores de la salud y del riesgo del consumo problemático, y la identificación de elementos socioculturales para la proyección de una propuesta preventiva localizada del consumo problemático de alcohol.

\section{METODOLOGÍA}

La metodología empleada para llevar a cabo esta investigación fue cualitativa, con un diseño emergente sobre la base de la lógica de la teoría fundamentada. La información fue producida a través de trabajo etnográfico de campo y de entrevistas en profundidad.

El trabajo de campo fue realizado en la costa lafkenche de la región de la Araucanía, distante a 30 kilómetros de Carahue, centro urbano más próximo, en las localidades de Coi-coi, Bajo Yupehue y Hueñalihúen, habitadas por una población mayoritariamente mapuche lafkenche. El acceso a la realidad etnográfica, se realizó durante los años 20162017, a partir de la observación participante en tres talleres realizados en los centros de salud rurales de cada una de las comunidades focalizadas, en estos el foco estuvo puesto en la comprensión del punto de vista de los actores sociales sobre el consumo 
problemático de alcohol y sus implicancias individuales, familiares y comunitarias. Durante este periodo se realizaron nueve entrevistas en profundidad en la modalidad relatos de vida $^{(35)}$ a personas adultas, siete varones y dos mujeres adscritas a la identidad cultural mapuche y habitantes de localidades rurales de la costa de la comuna de Carahue. Las entrevistas realizadas a varones se configuraron de la siguiente forma: dos realizadas a consumidores problemáticos, dos a personas recuperadas del consumo problemático y tres a personas no consumidoras de alcohol. Esta categorización inicial permitió obtener una mirada comparativa entre la relación con el alcohol y la identidad cultural en las trayectorias de vida de los sujetos.

Las entrevistas se orientaron a configurar relatos de vida en relación con el contexto familiar, comunitario y sociocultural. La técnica del relato de vida, permite descubrir lo cotidiano, las prácticas de vida dejadas de lado o ignoradas por las miradas dominantes, la historia de y desde los de abajo, a su vez, se destaca la importancia de la perspectiva del individuo como punto de observación de la sociedad en general ${ }^{(36)}$. Para la configuración de la muestra, se utilizó un tipo de muestreo teórico, por medio del cual se seleccionaron casos a estudiar según su potencial para ayudar a refinar o expandir los conceptos desarrollados ${ }^{(37)}$.

La información producida en las entrevistas fue sometida a un análisis cualitativo con el software ATLAS.ti (versión 6.0). Se realizó triangulación por informante al contar con la visión de más de una persona por dimensión a investigar y triangulación por expertos. En relación con los aspectos éticos de la investigación, una vez contactadas las comunidades y las personas a entrevistar, se informó sobre los objetivos y procedimientos de la investigación y fueron aplicados protocolos de consentimiento informado aprobados por el Comité de Ética Científica de la Universidad de la Frontera (Acta de aprobación n099, emitida el 16 de noviembre de 2016).

\section{RESULTADOS}

Los resultados de la investigación se organizan en tres partes: la primera referida a la construcción local sobre el riesgo y lo problemático del consumo. Una segunda, se relaciona con la identificación de escenarios y procesos protectores y de riesgo del consumo de alcohol que emergen del análisis comparativo de las trayectorias y relatos de vida de los sujetos entrevistados. Y, en una tercera parte, se identifican elementos socioculturales que orientan la proyección de una estrategia preventiva del consumo de alcohol y promocional de la salud.

\section{Construcción local del riesgo y patrones de iniciación, mantención y agravamiento}

El análisis de las trayectorias de vida de los sujetos entrevistados, indica un inicio temprano en el consumo de alcohol en la niñez y en la adolescencia. En la niñez se encuentra asociado a una baja supervisión parental y a la permisibilidad del consumo de alcoholes fermentados (chicha, vino) en faenas agropecuarias, en cambio, en la adolescencia, los espacios de iniciación y mantención del consumo tendrían un mayor grado de validación y normalización social en contextos de sociabilización familiar, laboral y comunitaria.

La mantención de los patrones de consumo problemático se sostiene en las formas de sociabilidad comunitaria y de integración productiva-laboral, que significan al consumo como espacio de encuentro, intercambio y de respuesta a las necesidades de reproducción de la vida cotidiana. Esto queda reflejado en el siguiente relato:

Por ejemplo, hay una siembra de papas, todos los ayudantes van ahí porque va a correr blanco o tinto, siquiera chicha, van a cooperar y ese es el medio de pago, entonces se va a consumir, pero al día siguiente no se puede seguir haciendo lo mismo, pero sin embargo pasa eso. (Pr1) 
Respecto de los significados en torno al consumo, se desprende que lo problemático tiende a homologarse con la significación de consumo de gravedad y dependencia al alcohol, se asocia con la pérdida de funcionalidad para sobrellevar la cotidianeidad estructurada sobre su base productiva de sustento diario para sí mismo y las personas que dependen del sujeto consumidor. En la Figura 1 se esquematiza la relación entre las fases que conforman las trayectorias de desarrollo del consumo problemático de alcohol (iniciación, mantención, agravamiento y dependencia) y la normalización del consumo, configurándose como indicador cultural de riesgo la pérdida de funcionalidad vinculada al desarrollo de dependencia al alcohol.

\section{Escenarios y procesos de riesgo y protectores del consumo problemático de alcohol}

La distinción entre procesos y escenarios de riesgo, y protectores que surgen del análisis pueden organizarse en dos perspectivas: la primera describe aspectos diacrónicos y transgeneracionales implicados en la conformación de los escenarios de riesgo y protección y, la otra, describe de manera sincrónica los significados del riesgo y la protección construidos y actualizados en los distintos contextos comunitarios de interacción significativa.

Los escenarios de riesgo aluden a contextos situacionales cotidianos y recurrentes que forman parte de los modos y estilos de vida de los sujetos y comunidades, y se vinculan con ámbitos de tipo laboral, comunitario y familiar. Uno de estos escenarios es la migración por trabajos agrícolas de temporada a regiones de la zona central del país, la que se asocia con la iniciación en el consumo y la adquisición de patrones de consumo problemático. En dichos contextos se transan subjetividades y son apropiados patrones y lógicas de consumo foráneos, los que, a su vez, se extrapolan a las dinámicas locales, resignificando los ritmos productivos, los periodos de ociosidad y desocupación, y las formas de sociabilidad. Así lo expresa uno de los entrevistados:

Sí, llegaba acá de repente para las fiestas, aquí tomaba porque con lo del trabajo tenía todos los días libres, y salía con los amigos a tomar. [...] Y allá era distinto, porque después tenían que ir a trabajar. [...] Si poh, por eso mismo sábado y domingo no más. (Pr2)

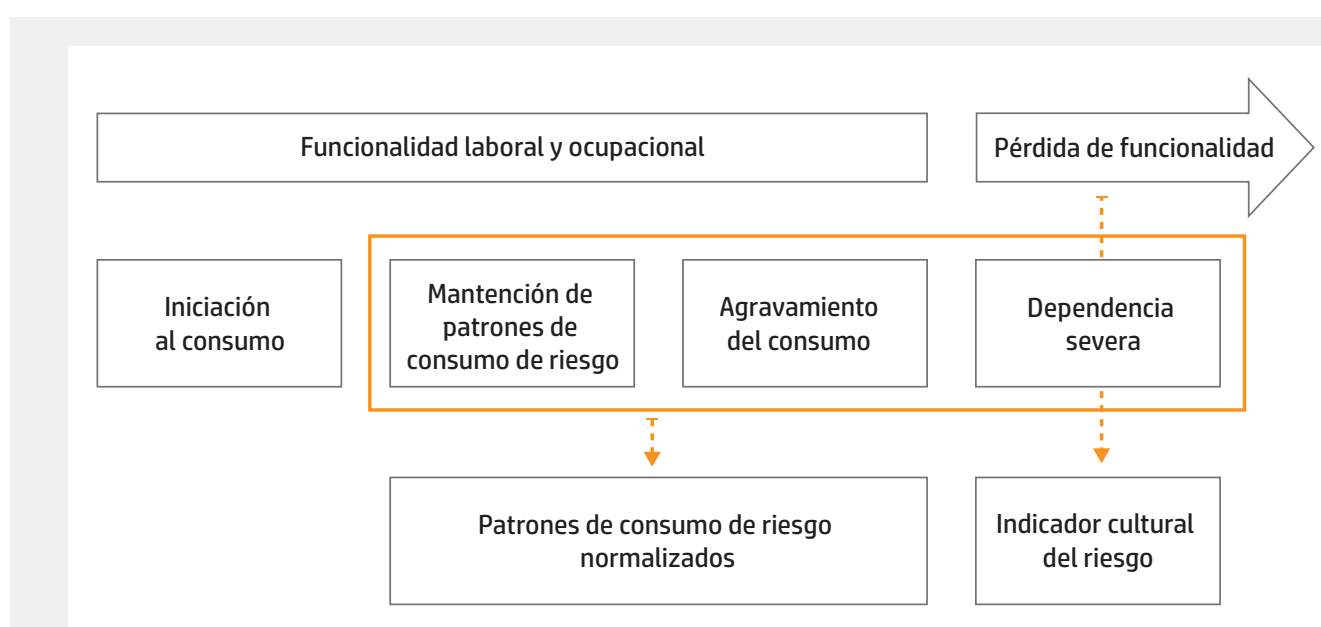

Figura 1. Trayectoria de consumo e indicador cultural del riesgo. 
El consumo tradicional, como escenario de riesgo, alude a la interacción histórica entre el saber occidental y aquel "saber menos

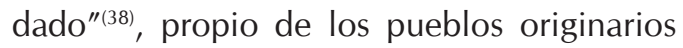
y del pueblo mapuche, respecto del uso de sustancias que alteran los estados de conciencia. En las entrevistas se mencionaron bebidas alcohólicas fermentadas, el muday de maíz, de chupón y una sustancia alucinógena llamada "miyaya". Si bien los usos culturales tradicionales de estas sustancias, en el contexto de los procesos de transculturación, han sido debilitados y perdidos, aún pervive esta producción y consumo de alcohol tradicional en la fabricación del muday de arveja, de maíz y la chicha de manzana, en la ritualidad ceremonial y en las pautas de sociabilidad y comensalidad que genera el consumo. De acuerdo a lo reportado en las entrevistas, en la cultura mapuche tradicional la alteración de los estados de conciencia en los niños a través del suministro de miyaya no era percibida y/o significada como nociva, el uso se daba en contextos específicos y al alero de un sistema de creencias sobre la formación de la persona ${ }^{(39)}$. Un entrevistado lo relata de la siguiente forma:

La miyaya, decía mi madre siempre, que le daban pa' probar como iba a ser el niñito, le daban chiquitito, muy poco de miyaya era como una droga así, para probar como su forma de cómo iba a ser cuando grande, y dice la historia, no de libros sino de la gente, que de repente se le pasaba la mano de quienes les daba y quedaban con problemas de salud mental y a ellos le llamaban miyaya kuntre, que se le pasó la mano, le dieron mucha dosis. (Pr1)

El consumo tradicional de bebidas fermentadas, conforma un escenario de riesgo en la medida en que estas prácticas o ciertos aspectos que persisten de ellas, se actualizan en espacios de interacción cotidiana desprovistas de sentidos y marcos regulatorios del consumo que lo restringían y vinculaban a espacios, momentos y significados culturales específicos.
A su vez, el contexto familiar configura escenarios de riesgo para el desarrollo del consumo problemático de alcohol en situaciones tales como la cercanía de un familiar con dependencia, que influye en la iniciación y/o el mantenimiento de patrones de consumo problemáticos, por la proximidad de la apertura del espacio de interacciones, acciones y significados que posibilitan y mantienen el consumo, desde la obtención y acceso al producto, hasta la red de relaciones y escenarios de riesgo donde este consumo se desarrolla. Así también la ausencia de una persona significativa, de comunicación familiar orientadora para la vida y preventiva del consumo de alcohol. En palabras de un entrevistado con consumo:

Ellos nunca nos contaron eso... nada de eso [...] no nos enseñó a nosotros. Nosotros no tuvimos consejos de mi padre, nada, nada, ni de mi mamá. Nosotros no tuvimos un consejo de nuestro padre como le digo, si hubiese vivido en el pueblo no sé cómo hubiese sido. Si no hubiésemos vivido en el campo no sé cómo habríamos sido. (Pc1)

El consumo de alcohol, entonces, constituye un dispositivo de sociabilización, de apertura a compartir con otros, respondiendo a una necesidad social y simbólica de los sujetos. En este escenario, adquiere un carácter coercitivo, en el cual los sujetos son inducidos e inductores al consumo en el contexto de grupo, en el que estaría ausente la reflexión colectiva sobre lo problemático del consumo. Se produce además una discontinuidad intersubjetiva con el espacio íntimo-domésticofamiliar al omitir y evadir las implicancias e impacto de este consumo en esos espacios. La ausencia de apoyo que reportan los entrevistados, en algunos casos desde las propias familias y de la comunidad, se traduce en una ausencia de respuestas organizadas y colectivas frente al problema, lo que cierra el círculo del riesgo del beber, problema ligado a los espacios de interacción locales. Así lo manifiesta un entrevistado recuperado del consumo: 
...me van a decir que para qué estoy haciendo esto, tomemos no más, entonces yo prefiero hacerlo solo, porque si yo lo hago con ellos, ellos en vez de ayudarme no me van a ayudar, me van a decir no que andas haciendo eso, va a ser para peor, tomemos mejor, va a ser lo primero que me van a decir, y estoy seguro que eso me van a decir porque yo lo he visto y varias veces que cuando yo no quiero tomar ellos me exigen. (Pc2)

\section{Transculturación como proceso de riesgo}

Las comunidades mapuches del sector costero de la Araucanía, han experimentado procesos de transculturación históricos, lo que se expresa a nivel sociocomunitario, familiar e individual. Este proceso, se expresa en las trayectorias de vida de los sujetos entrevistados, a través de aspectos tales como: el desconocimiento de la cultura y la lengua, así como en las vivencias de discriminación, estigmatización y violencia simbólica, tanto por origen étnico-cultural, como por la condición de "ser pobres" y "campesinos" que, según reportan los entrevistados, en su niñez, habría sido recurrente en el ámbito escolar, por parte de profesores y compañeros, adquiriendo nuevas formas y matices en etapas biográficas posteriores. Estas situaciones inciden en la dificultad de asumir la identidad mapuche, en el desinterés y apatía hacia la cultura originaria y/o una valoración funcional desprovista de sus dimensiones simbólicas para la obtención de beneficios transferidos a través de políticas públicas.

Otro aspecto relevante de estos procesos de transculturación es la pérdida y debilitamiento de normativas regulatorias de la vida cotidiana en general y del consumo de alcohol en particular, proceso que se retroalimenta con la deslegitimación de las autoridades tradicionales en el sector, y que es reforzado por la difusión y adopción del sistema de creencias cristiano-evangélico pentecostal en las comunidades mapuche locales.
Cabe señalar que la introducción histórica del alcohol en estas comunidades rurales formó parte de dispositivos de dominación utilizados en el marco de la ocupación de los territorios mapuche, y es un aspecto emergente en el análisis de los datos. Es posible inferir que la historia de estas comunidades estuvo atravesada por hechos y episodios violentos y traumáticos, en los que el consumo de alcohol tuvo un papel significativo. Un entrevistado reporta lo siguiente:

Se hicieron amigos, pero la amistad era
con interés, hicieron una estrategia, bus-
caron quienes eran los lonkos, las perso-
nas más importantes de esa comunidad.
Mandaron a hacer un caserón grande,
como un galpón, y dice que mataron
animales, trajeron vino y todo, y, hicie-
ron un banquete, o como un mizagun
diría el mapuche, y dice que una vez que
estaban todos curados, así como, cura-
dos, entre familia, todo, y niños desde
todas edades, dice que este mandó a
sus mocetones, a sus camperos serían,
no sé [...] y dice que le prendió fuego
adentro... y los quemó a todos... y eso
ocurrió acá en el sector de más abajo
de Pilolcura. Esa comunidad se llamaba
Tricahuera, y ahora esa comunidad no
existe [...] a mí me contó el lonko [...],
y eso ocurrió a través del alcoholismo
igual. (Pnc1)

Estos antecedentes reportan la relación histórica entre el alcohol y procesos de transculturación experimentados por las comunidades locales, en los que se configura una memoria colectiva descontinuada y nebulosa, un espacio intersubjetivo difuso cuya evocación pareciera evitarse, como parte de los síntomas del trauma intergeneracional de la comunidades mapuche costeras de la Araucanía ${ }^{(27)}$.

A modo de síntesis, los procesos y escenarios de riesgo analizados se relacionan con múltiples aspectos subjetivos, dinámicas familiares y comunitarias que interactúan de manera sinérgica y se expresan en las trayectorias de vida de los sujetos. En la Tabla 1 se expone una síntesis que los vincula a las 
distintas fases del desarrollo del consumo problemático de alcohol.

\section{Procesos y escenarios protectores}

Los escenarios protectores que emergen de los datos están vinculados de forma exclusiva al contexto familiar: la comunicación afectiva y orientadora para la vida y la regulación del consumo de alcohol influyen en la formación de personas capaces de resistir la adquisición de patrones de consumo problemático normalizados en el medio social comunitario. Otro escenario protector que aumenta la percepción del riesgo del consumo de alcohol, es la experiencia de un familiar cercano con dependencia, lo que se traduce en un ejemplo para no replicar en la propia vida, así lo expresa un entrevistado no consumidor:

El alcohol aquí es un factor $\mathrm{mmm}$, yo lo veo muy malo para la gente y sobre todo para la gente que vende, porque lo tuve un problema con mi hermano, yo lo veía mal, estuvo muy mal, a donde vaya invitan a tomar, por eso cuando yo era chico decía que no iba a ser así. (Pr2)
Además del escenario familiar, se identifican dos procesos protectores, el primero estaría relacionado con la presencia de las iglesias evangélicas que ofrecen un espacio comunitario de contención espiritual y emocional a las personas que padecen consumo problemático y participan en estos. Algunos entrevistados señalan que estas iglesias con presencia creciente en el sector, tendrían incidencia positiva en la rehabilitación de personas con consumo. La siguiente cita de un entrevistado no consumidor alude a lo anterior:

Hay gente que, estaban sumidas en el alcohol, sumidas, pero así, ya nadie daba un peso en la vida de esas personas, y, increíble que se han rehabilitado [...] Se han rehabilitado $y, y$ ha sido un cambio, pero increíble. (Pnc1)

El segundo proceso protector es la valoración personal y colectiva de la identidad cultural mapuche, que alude a la vitalidad cultural como actualización en la vivencia de los valores y costumbres propios de la cultura y se relaciona con la conciencia individual y colectiva de la propia identidad, las formas tradicionales de vivir en comunidad y transmitir valores, sistema de creencias y los marcos

Tabla 1. Síntesis de procesos y escenarios de riesgo del consumo de alcohol.

\begin{tabular}{|c|c|c|c|c|}
\hline Identidad cultural & $\begin{array}{l}\text { Iniciación en el } \\
\text { consumo en la } \\
\text { infancia }\end{array}$ & $\begin{array}{l}\text { Iniciación en el } \\
\text { consumo en la } \\
\text { adolescencia }\end{array}$ & $\begin{array}{l}\text { Mantención del } \\
\text { consumo }\end{array}$ & $\begin{array}{l}\text { Agravamiento } \\
\text { del consumo y } \\
\text { dependencia }\end{array}$ \\
\hline $\begin{array}{l}\text { Pérdida cultural hogar } \\
\text { de origen }\end{array}$ & $\begin{array}{l}\text { Baja supervisión } \\
\text { parental. Tolerancia en } \\
\text { contexto productivo }\end{array}$ & $\begin{array}{l}\text { Pertenencia a grupos de } \\
\text { pares, coerción grupal }\end{array}$ & $\begin{array}{l}\text { Ausencia de } \\
\text { problematización }\end{array}$ & $\begin{array}{l}\text { Pérdida de vínculos } \\
\text { familiares }\end{array}$ \\
\hline $\begin{array}{l}\text { Pérdida cultural } \\
\text { comunidad de origen }\end{array}$ & $\begin{array}{l}\text { Distancias } \\
\text { comunicativas } \\
\text { familiares }\end{array}$ & $\begin{array}{l}\text { Tolerancia social al } \\
\text { consumo }\end{array}$ & $\begin{array}{l}\text { Coerción grupal al } \\
\text { consumo }\end{array}$ & Abandono familiar \\
\hline $\begin{array}{l}\text { Desvaloración identidad } \\
\text { cultural }\end{array}$ & $\begin{array}{l}\text { Baja supervisión de } \\
\text { los padres, adultos } \\
\text { cuidadores }\end{array}$ & $\begin{array}{l}\text { Migración temporal al } \\
\text { norte }\end{array}$ & $\begin{array}{l}\text { Desvinculación } \\
\text { comunitaria }\end{array}$ & $\begin{array}{l}\text { Pérdida de funcionalidad } \\
\text { fissica. Déficit } \\
\text { ocupacional }\end{array}$ \\
\hline $\begin{array}{l}\text { Valoración funcional de } \\
\text { la cultura }\end{array}$ & $\begin{array}{l}\text { Curiosidad/ } \\
\text { experimentación }\end{array}$ & $\begin{array}{l}\text { Espacio de } \\
\text { sociabilización }\end{array}$ & Desvinculación familiar & \\
\hline $\begin{array}{l}\text { Discriminación, estigma, } \\
\text { exclusión social }\end{array}$ & $\begin{array}{l}\text { Consumo problemático } \\
\text { de alcohol en el hogar }\end{array}$ & $\begin{array}{l}\text { Consumo problemático } \\
\text { de alcohol en el hogar }\end{array}$ & $\begin{array}{l}\text { Ausencia de apoyo } \\
\text { familiar y comunitario }\end{array}$ & \\
\hline $\begin{array}{l}\text { Debilitamiento de la } \\
\text { memoria colectiva }\end{array}$ & $\begin{array}{l}\text { Bebidas fermentadas } \\
\text { (chicha, muday, pulque) } \\
\text { como alimento }\end{array}$ & $\begin{array}{l}\text { Bebidas fermentadas } \\
\text { (chicha, muday, pulque) } \\
\text { como alimento }\end{array}$ & & \\
\hline
\end{tabular}


regulatorios del comportamiento. Esta valoración es transmitida, principalmente, por la familia y en menor grado por la comunidad, y se expresa a nivel individual en aspectos tales como la conciencia de la propia identidad y la reflexión identitaria como parte de la formación de la persona. Lo que refieren en común estos entrevistados es haber vivenciado un proceso a través del cual han asumido su identidad cultural con aceptación, denotando bajos niveles de negación, invisibilización o desvalorización en sus discursos. Resulta significativo que estos sujetos pertenezcan a la categoría de no consumidores y recuperados del consumo:

\begin{abstract}
No, siempre o sea a mí siempre me preguntaban mi apellido y yo siempre lo decía y nunca lo di con vergüenza, siempre dije que era de acá de Temuco, Carahue y nunca me avergoncé de nada, yo usé el pelo largo y me faltaba el puro cintillo [risas]. (Pnc2)
\end{abstract}

La reflexión identitaria, como se presenta en los discursos de los entrevistados, expresa la vivencia de un proceso que fortalece la valoración de lo propio. Un entrevistado recuperado del consumo de alcohol lo señala:

\begin{abstract}
...igual he tenido que decirle a la gente que dice que nosotros respetamos la cultura del pueblo mapuche y yo le pregunto ¿de qué forma nos respetan cuando el día sábado nos hablan de un Cristo crucificado, y el día domingo hablan de otro Cristo que por ese Cristo están vivos?... y ¿es respeto eso? Quedan calladitos, porque esa son formas de sobreponerse al conocimiento de un pueblo. $(\operatorname{Pr} 1)$
\end{abstract}

La Tabla 2, muestra una síntesis que vincula escenarios y procesos protectores con los contextos relacionales de la familia y de la comunidad. La valoración identitaria es un proceso protector transversal en estos contextos.

Se concluye en este apartado que los escenarios protectores del consumo problemático de alcohol se sitúan de forma exclusiva en el contexto familiar, y que los procesos protectores de conversión evangélica y vitalización de la cultura mapuche se encontrarían en posiciones contrapuestas. Se advierte que la iglesia evangélica como espacio de cohesión y contención afectiva-emocional estaría en tensión con los procesos de valoración y conciencia de la identidad cultural, toda vez que, según lo declarado por los sujetos participantes de este estudio, el sistema de creencias y dogma evangélico pentecostal tiende a excluir al sistema de creencias y prácticas rituales-ceremoniales mapuche. Lo anterior implica que la comprensión y abordaje de esta complejidad requiere distinciones analíticas que trascienden y cuestionan la categorización dicotómica y simplificadora de protección y de riesgo de los procesos enunciados. La Figura 2 grafica esta situación en la que ambos procesos considerados protectores se contraponen y tensionan.

El análisis de contrastación de las trayectorias de vida de las personas entrevistadas que presentan consumo problemático de alcohol, recuperadas del consumo y no consumidoras (Tabla 3), indica que las primeras señalan mayor distancia comunicativa con su medio familiar y una menor participación en organizaciones sociales y comunitarias.

Tabla 2. Escenarios y procesos protectores y vinculación familiar y comunitaria.

\begin{tabular}{|c|c|c|c|}
\hline $\begin{array}{l}\text { Identidad } \\
\text { cultural }\end{array}$ & $\begin{array}{l}\text { Vínculo } \\
\text { familiar en la } \\
\text { infancia }\end{array}$ & $\begin{array}{l}\text { Vínculo } \\
\text { familiar en la } \\
\text { adolescencia }\end{array}$ & $\begin{array}{l}\text { Vínculo } \\
\text { comunitario }\end{array}$ \\
\hline $\begin{array}{l}\text { Vitalidad } \\
\text { cultural en hogar } \\
\text { y comunidad de } \\
\text { origen }\end{array}$ & $\begin{array}{l}\text { Supervisión } \\
\text { parental }\end{array}$ & $\begin{array}{l}\text { Pertenencia } \\
\text { a grupos } \\
\text { de pares no } \\
\text { consumidores }\end{array}$ & $\begin{array}{l}\text { Participación } \\
\text { comunitaria } \\
\text { selectiva }\end{array}$ \\
\hline $\begin{array}{l}\text { Valoración de } \\
\text { la identidad } \\
\text { cultural }\end{array}$ & $\begin{array}{l}\text { Comunicación } \\
\text { afectiva familiar }\end{array}$ & $\begin{array}{l}\text { Regulación del } \\
\text { consumo en el } \\
\text { hogar }\end{array}$ & $\begin{array}{l}\text { Pertenencia a } \\
\text { organizaciones } \\
\text { prosociales }\end{array}$ \\
\hline $\begin{array}{l}\text { Traspaso } \\
\text { de valores } \\
\text { y normas } \\
\text { regulatorias del } \\
\text { comportamiento }\end{array}$ & $\begin{array}{l}\text { Regulaciones } \\
\text { del consumo en } \\
\text { hogar de origen }\end{array}$ & $\begin{array}{l}\text { Autoestima } \\
\text { fortalecida }\end{array}$ & $\begin{array}{l}\text { Pertenencia a la } \\
\text { iglesia cristiana } \\
\text { evangélica }\end{array}$ \\
\hline $\begin{array}{l}\text { Conciencia } \\
\text { de identidad } \\
\text { y reflexión } \\
\text { identitaria }\end{array}$ & $\begin{array}{l}\text { Conocimiento } \\
\text { familiar del } \\
\text { riesgo del } \\
\text { desarrollo de } \\
\text { dependencia }\end{array}$ & & \\
\hline
\end{tabular}




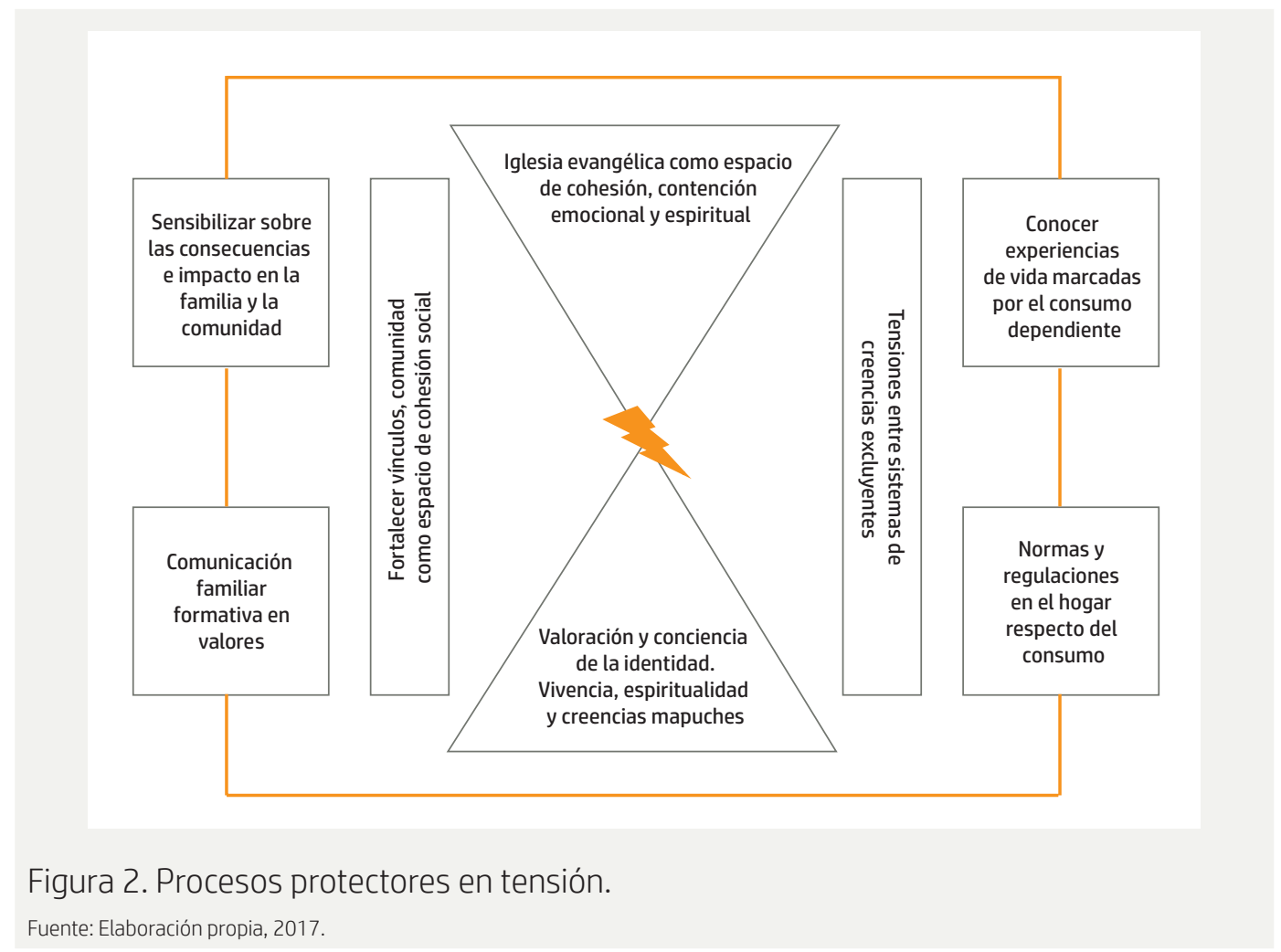

Por otro lado, muestran mayor desconocimiento y desvalorización de la dimensión ideacional-simbólica de la cultura, y una valoración funcional de esta asociada a la transferencia institucional de recursos y reivindicación de derechos de tierra. Diferente es el caso de las personas recuperadas del consumo, quienes declaran mayor apego a la familia de origen y/o constituida, y una mayor vinculación a organizaciones sociales y comunitarias locales. Este grupo muestra un proceso de reflexión y valoración en torno a la identidad cultural mapuche en sus trayectorias de vida. A su vez, en este grupo hay un modelamiento y selectividad en la vinculación a contextos de sociabilización, que dis-

Tabla 3. Contrastación de trayectorias de vida, valoración de la identidad cultural y vinculación a contextos de interacción.

\begin{tabular}{lllll}
$\begin{array}{l}\text { Trayectorias de } \\
\text { vida }\end{array}$ & $\begin{array}{l}\text { Vinculación } \\
\text { familiar }\end{array}$ & Vinculación comunitaria & $\begin{array}{l}\text { Valoración } \\
\text { identidad } \\
\text { cultural }\end{array}$ & Observaciones \\
$\begin{array}{l}\text { Con consumo } \\
\text { problemático }\end{array}$ & $\begin{array}{l}\text { Distancia } \\
\text { comunicativa }\end{array}$ & $\begin{array}{l}\text { Sin participación, un caso } \\
\text { en organización mapuche } \\
\text { territorial }\end{array}$ & $\begin{array}{l}\text { Desvalorización } \\
\text { y valoración } \\
\text { funcional de la } \\
\text { cultura }\end{array}$ & $\begin{array}{l}\text { Ausencia de valoración de } \\
\text { significados y cosmovisión } \\
\text { mapuche. Debilitamiento } \\
\text { vinculación comunitaria }\end{array}$ \\
$\begin{array}{l}\text { Recuperadas del } \\
\text { consumo }\end{array}$ & Apego familiar & Iglesia evangélica y laboral & Valoración & $\begin{array}{l}\text { Modelamiento vinculación } \\
\text { con el medio a partir de } \\
\text { proceso recuperativo }\end{array}$ \\
No consumidoras & Apego familiar & $\begin{array}{l}\text { Iglesia evangélicay y } \\
\text { organización comunitaria } \\
\text { vecinal }\end{array}$ & Valoración & $\begin{array}{l}\text { Vinculación positiva a } \\
\text { contextos y valoración de } \\
\text { la identidad cultural }\end{array}$ \\
\hline Fuente: Elaboración propia, 2017. & & & \\
\hline
\end{tabular}


minuyen la participación y el vínculo en espacios recreativos, y los fortalecen en espacios laborales, familiares y/o religiosos. Por último, el grupo de las personas que no presentan consumo de alcohol muestran una vinculación positiva a contextos familiares y comunitarios, una mayor adscripción en organizaciones comunitarias: juntas de vecinos, comunidades indígenas y/o participación en las iglesias locales. A su vez, este grupo declara mayor conocimiento, cercanía y vivencia de la cultura mapuche desde sus hogares y comunidades de origen.

La Tabla 3 muestra una síntesis que relaciona las distintas categorías de entrevistados con el tipo de vínculo con el contexto familiar, comunitario y la valoración de la identidad cultural.

\section{Orientaciones para la proyección de una estrategia preventiva del consumo de alcohol y promocional de la salud}

Los aspectos a considerar en la proyección de actuaciones preventivas pueden ser englobados en tres ámbitos: preventivo/promocional, rehabilitación y de fortalecimiento comunitario; a su vez, se realizan algunas distinciones para la intervención con sujetos, familia y comunidad. La valoración de la identidad cultural es un aspecto transversal que atraviesa estos ámbitos y niveles de intervención.

Se advierte que, para actuar sobre el marcador cultural del riesgo, es necesario problematizar a nivel comunitario la noción de consumo problemático a partir de su implicación con otros aspectos de la vida y la convivencia, tales como la familia, la formación de los hijos, la comunicación familiar, la relación entre vecinos, entre otros.

Para actuar de manera preventiva sobre la iniciación en el consumo en la infancia y en la adolescencia se sugiere, por un lado, problematizar la construcción social y local del riesgo a la luz de la evidencia científica; informar y educar sobre el fenómeno neurobiológico del consumo en esta etapa vital y, por otro lado, afianzar procesos previos de fortalecimiento de la identidad personal y cultural de los jóvenes, a partir de acciones familiares y comunitarias. En este marco, se sugiere la activación de procesos colectivos de intraculturación, que aluden al derecho a ser educado sobre la base de la cultura propia $^{(40)}$ y al conocimiento y valoración cultural y social propia, a través de la complejidad y la diferencia interna del propio grupo social para abarcar aspectos interculturales y de relación desde la equidad con otras culturas $^{(41)}$. Se estima necesario, entonces, abordar la identidad cultural a nivel comunitario y familiar desde una perspectiva dialógica, socioeducativa y de sociopraxis, de modo de disparar procesos de reflexión, problematización y análisis de la actual situación de estas comunidades. En este proceso cobra relevancia la activación de acciones de recuperación de la memoria colectiva e histórica, un acercamiento comprensivo a la cosmovisión mapuche y los valores de formación de la persona en la cultura mapuche (chengen).

En relación con los procesos protectores tensionados, se estima necesaria la apertura de un espacio donde las distintas visiones e imaginaciones colectivas puedan encontrarse y dialogar sobre temas relacionados con la prevención y rehabilitación del consumo de alcohol, y las distintas violencias asociadas, desde una perspectiva integral, que considere los distintos aspectos involucrados en el problema a nivel local. Un espacio, en el que evaluar convergencias y divergencias en el desafío de construir un piso ético-valórico común, desde donde puedan surgir consensos, es decir, poner en la mesa estas tensiones con el propósito de que emerjan soluciones desde las propias comunidades. En esta línea se sugiere definir y anclar procesos y actuaciones en contextos comunitarios locales: centros de salud, escuelas, juntas de vecinos, organizaciones sociales de base del sector.

Respecto de las acciones orientadas a rehabilitar personas, el abordaje centrado en el sujeto que se realiza desde los dispositivos de salud locales resulta insuficiente para intervenir de manera efectiva en el problema, ya que la intervención clínica proyecta un proceso de recuperación vivenciado en solitario, lo que se traduce en que la persona que 


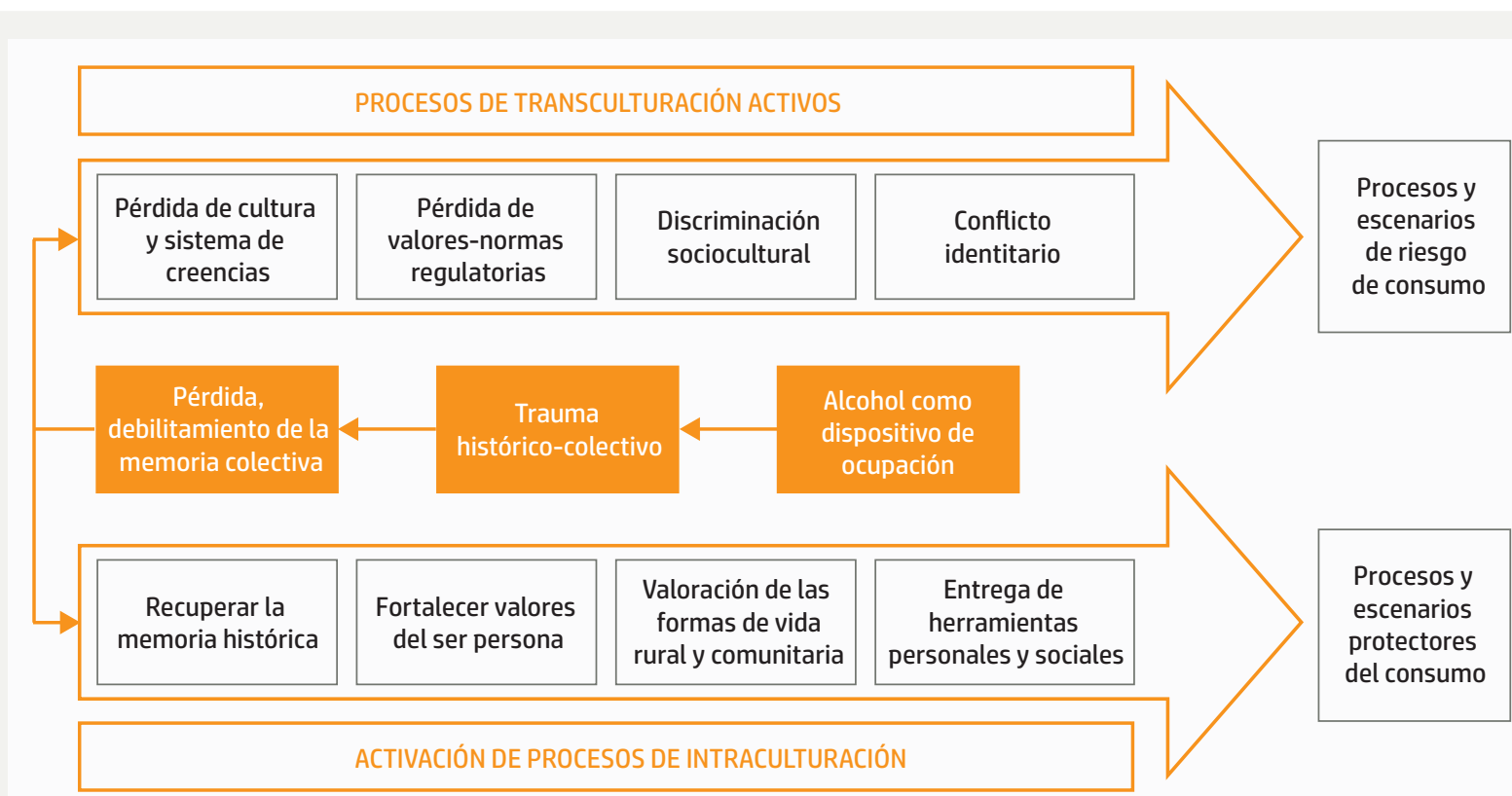

Figura 3. Contraposición de procesos protectores por activar y de riesgo activos.

Fuente: Elaboración propia, 2017.

accede al tratamiento, debe tomar distancia de los espacios de participación familiar y comunitarios en los que el consumo opera como dispositivo de sociabilidad, quedando subjetivamente aislado de su entorno y desprovisto de apoyos para mantenerse sin consumo. Todo ello implica, en muchos casos la deserción al tratamiento y la recaída. Ante estos antecedentes, se propone un abordaje amplificado a los niveles interaccionales familiar y comunitario, una intervención ecológica que incorpore acciones tendientes a configurar entornos sociales de acompañamiento al sujeto en recuperación, a partir de acciones de problematización y concienciación familiar y comunitaria, con el propósito de fortalecer la adherencia y la eficacia del tratamiento. Estos procesos de intervención ecológica en el contexto de vida de los sujetos con consumo problemático, tienen un doble valor y función al transitar entre los espacios de rehabilitación y prevención/promoción de la salud.

Desde el conocimiento social local, el problema del consumo abusivo de alcohol es percibido como consecuencia del abandono en que se encuentran las comunidades del sector por parte de las políticas públicas, a las cuales se les atribuye responsabilidad por la falta de trabajo, se señala que el problema del alcoholismo no sería el más importante que les aqueja, sino la falta de empleo, la ociosidad en que se encuentran los adultos en edad productiva. Desde el punto de vista de los informantes clave, esta percepción reflejaría el debilitamiento y la pérdida cultural, se considera que la demanda de trabajo al Estado representaría una contradicción con la cultura y formas de vida mapuche, así como la ausencia de una conciencia y posicionamiento político ante procesos neocoloniales. En este punto resulta reveladora la tendencia a la pasividad de estas personas y colectividades, su mirada de la institucionalidad como un mecanismo de transferencia de conocimientos y recursos que adoptan o desechan, de acuerdo a la ponderación de sus propias valoraciones, todo ello sugiere la necesidad de activar procesos de fortalecimiento del tejido comunitario.

En la Figura 3 se expone de manera ilustrativa la contraposición de procesos de transculturación como proceso de riesgo para el consumo de alcohol y la promoción 
de procesos protectores de valoración y vitalización cultural.

\section{DISCUSIÓN Y CONCLUSIONES}

El análisis de las trayectorias de vida y la observación participante con estas comunidades mapuche costeras de la Araucanía muestran que el debilitamiento y la desvalorización de la identidad cultural se configuran como un proceso de riesgo al interactuar con otros escenarios y procesos de riesgo en la iniciación, mantención y agravamiento del consumo de alcohol. Al respecto, es importante mencionar que la vinculación de los procesos de transculturación con el consumo problemático de alcohol, no aparece de forma explícita en los discursos de los entrevistados, sino en la clave cultural que naturaliza e invisibiliza procesos: la pérdida cultural expresada en una baja identificación y valoración cultural, en el debilitamiento de la memoria colectiva y en la baja cohesión social y comunitaria. Según ha sido analizado, en el contexto local coexisten sistemas de creencias que, si bien de forma separada conforman procesos protectores del consumo de alcohol, en su dinamismo y convivencia entran en conflicto: las comunidades cristianas pentecostales tienden a acelerar procesos de transculturación y pérdida cultural, es decir, al tensionar la activación de procesos de valoración de la cultura mapuche, estaría configurando un proceso de riesgo.

Esto coincide con lo reportado en un estudio previo realizado con autoridades tradicionales mapuches ${ }^{(42)}$, en el cual se reconoce al alcohol como un desorganizador cultural, a la vez que un síntoma de esta desorganización.

Resultados similares, que vinculan dinámicas de transculturación y pérdida cultural con la emergencia de problemas de salud mental y consumo problemático de sustancias han sido reportados en contextos culturales australianos, estadounidenses y otros países de Latinoamérica ${ }^{(33,43,44,45)}$. En este escenario complejo, se advierte que el abordaje preventivo del consumo de alcohol y promocional de la salud debería considerar estos aspectos. En esta línea, los hallazgos del proceso investigativo sustentan un cuestionamiento a la dicotomía de factores de riesgo y de protección que está en la base de las políticas preventivas, que tienden a simplificar y estandarizar la realidad invisibilizando la complejidad inherente a los fenómenos socioculturales, colectivos, intersubjetivos y transaccionales por naturaleza.

Las actuaciones interventivas que se desprenden de esta simplificación binaria no logran aprehender y abordar procesos históricos implicados en el problema, la pérdida de los marcos de referencia tradicionales, el cambio en los significados atribuidos al consumo, el debilitamiento de la cohesión comunitaria y estas tensiones o "verdades en disputa" que estarían en la base de estas dinámicas más o menos conflictivas de la vida sociocomunitaria.

Desde una mirada decolonial, la actual invisibilización de estos fenómenos socioculturales e intersubjetivos que se realiza desde las políticas e intervenciones preventivas tradicionales, reproducen realidades y saberes locales como no existentes ${ }^{(46)}$.

En esta perspectiva, la evidencia producida $^{(47)}$ advierte la necesidad de construir programas con pertinencia cultural que permitan acoger las complejidades antes señaladas; que la intervención se desarrolle a partir de los valores, creencias, prácticas y perspectivas sociohistóricas del grupo objetivo ${ }^{(48)}$, y recojan las fortalezas de la cultura construyendo desde el sentido de agencia, poder y lenguaje ${ }^{(49)}$, lo cual forma parte central de la estrategia.

En este contexto, los elementos socioculturales identificados proyectan un abordaje preventivo y promocional que considera actuaciones pensadas desde un enfoque dialógico y decolonial que pueden contribuir a un abordaje de fortalecimiento y empoderamiento comunitario para el desarrollo humano ${ }^{(50)}$. En esta línea, se advierte la necesidad de activar procesos de "conciencia de proceso" y sanación de la colectividad mediante el reconocimiento de la historia, 
la recuperación de la memoria colectiva, a partir del desarrollo de acciones de intraculturalidad, de valoración y fortalecimiento de la cultura ${ }^{(41)}$ en diálogo con las otras racionalidades interactuantes: institucional y teoría de la prevención basada en evidencia. En este punto, el análisis se conecta con la noción de trauma colectivo y transgeneracional acuñado por la psicología comunitaria canadiense y que junto con diagnosticar el trauma colectivo en las poblaciones que han padecido y padecen la/s violencia/s en sus múltiples facetas y dimensiones, abre la posibilidad de actuar sobre esas heridas colectivas y avanzar hacia su sanación. Para ello es necesario abordar de forma implicativa sentidos y significados locales históricamente construidos en la imaginación colectiva de un proyecto de mejora de las condiciones de vida.
La acción comunitaria tiene por propósito revitalizar la sociabilidad, aportando a la vinculación y organización social, como también a los emprendimientos colectivos para mejorar las condiciones de vida, en especial, de la población socialmente desfavorecida ${ }^{(51)}$. En esta perspectiva, el grupo y su organización aparece como un elemento que, dentro de un espacio social determinado, permite redensificar la vida social mediante el fortalecimiento del sujeto político ${ }^{(52,53)}$. De ahí entonces que los procesos de prevención y abordaje del alcoholismo supongan, a partir de los resultados, actuaciones desde una perspectiva comunitaria no colonial ${ }^{(54)}$, y la coordinación de servicios que se enlacen con las prioridades definidas por las propias comunidades.

\section{REFERENCIAS BIBLIOGRÁFICAS}

1. Organización Panamericana de la Salud. Alcohol y salud de los pueblos indígenas [Internet]. Washington DC: Oficina Regional para las Américas de la Organización Mundial de la Salud; 2005 [citado 1 jun 2018]. Disponible en: https:// tinyurl.com/y6b88zbj.

2. Organización Panamericana de la Salud. Promoción de la salud mental en las poblaciones indígenas: experiencias de países; Una colaboración entre la OPS/OMS, Canadá, Chile y Socios de la Región de las Américas 2014-2015 [Internet]. Washington DC: Organización Panamericana de la Salud; 2016 [citado 1 jun 2018]. Disponible en: https://tinyurl.com/yycdadkc.
3. Ministerio de Salud de Chile, Banco Mundial. Plan para pueblos indígenas: Proyecto de apoyo al sector salud [Internet]. Santiago de Chile: Ministerio de Salud; 2017 [citado 1 jun 2018]. Disponible en: https://tinyurl.com/y6x9nbvy.

4. Ministerio de Salud, Pontificia Universidad Católica de Chile. Informe final: Estudio de carga de enfermedad y carga atribuible. Ministerio de Salud [Internet]; 2008 [citado 1 jun 2018]. Disponible en: https://tinyurl.com/y4md4d2n.

5. World Health Organization. Global status report on alcohol and health-2014 [Internet]. Geneva: WHO; 2014 [citado 1 jun 2018]. Disponible en: https://tinyurl.com/yxavvwlk. 
6. Gobierno de Chile, Ministerio de Salud, Departamento de Epidemiología. Diagnósticos regionales con enfoque DSS. Santiago de Chile: Ministerio de Salud; 2012.

7. Observatorio Chileno de Drogas, Servicio Nacional de Prevención y Rehabilitación de Drogas y Alcohol. Décimo segundo estudio de drogas en población general de Chile, 2016 [Internet]. Santiago de Chile: Ministerio del Interior y Seguridad Pública; 2017 [citado 1 jun 2018]. Disponible en: https://tinyurl.com/y53282zb.

8. Ministerio de Desarrollo Social. Encuesta de caracterización socioeconómica nacional 2013 [Internet]. c2019 [citado 1 jun 2018]. Disponible en: https://tinyurl.com/y46elvmf.

9. Vial Cossani C. Índice de desarrollo regional: IDERE 2016 [Internet]. Santiago de Chile: Instituto Chileno de Estudios Municipales (ICHEM), Universidad Autónoma de Chile; 2016 [citado 1 jun 2018]. Disponible en: https://tinyurl.com/y2enf9ku.

10. Bengoa J. Historia del pueblo mapuche (siglo XIX y XX). Santiago de Chile: LOM Ediciones; 2000.

11. Muñoz Sougarret J. Notas sobre la relación entre alcohol y trabajo en la frontera sur de la Araucanía; Segunda mitad del siglo XIX. Vol.1. En: Fernández Labbé M, Godoy Sepúlveda E, Herrera González P, Muñoz Sougarret J, Venegas Valdebenito $\mathrm{H}$, Yáñez Andrade JC. Alcohol y trabajo: el alcohol y la formación de las identidades laborales; Chile Siglo XIX y XX. Osorno: Editorial Universidad de los Lagos; 2008. p. 37-63.

12. Oyarce A, Ñanco J. Alcoholismo y etnia, críticas y propuestas. En: Salgado MS, Mella IJ, (eds.). Salud, cultura y territorio: Bases para una epidemiología intercultural. Santiago de Chile: Ministerio de Salud; 1998. p. 35- 43.

13. Municipalidad de Carahue. Diagnóstico comunal sobre consumo de alcohol y otras drogas. Carahue: SENDA Previene; 2014.

14. Medina E. Aproximación antropológica al análisis epidemiológico: consideraciones sobre el modo de beber entre los mapuches. Cultura, Hombre, Sociedad. 1984;1(1):53-88. doi: 10.7770/ cuhso-V1N1-art131.

15. Berruecos L. El consumo de alcohol y el alcoholismo en México: el caso de las comunidades indígenas. El Cotidiano. 2013;28(181):73-80.

16. Duffy D, de Lellis M. Concepciones de los profesionales del sistema de salud sobre el consumo de alcohol. Anuario de Investigaciones. 2014;XXI: 207-215.
17. Margozzin P, Sapag J. El consumo riesgoso de alcohol en Chile: tareas pendientes y oportunidades para las políticas públicas. Temas de la Agenda Pública. 2015;10(75):1-15.

18. Menéndez E, Di Pardo R. Violencias y alcohol: Las cotidianidades de las pequeñas muertes. Vol. 19. Ciudad de México: CIESAS; 1998. p. 37-71.

19. United Nation Office on Drugs and Crime. World drug report; Executive summary: Conclusions and policy implications. UNODC [Internet] 2018 [citado 1 jun 2018]. Disponible en: https:// tinyurl.com/y4wlmguk.

20. Hersch-Martínez P. Epidemiología sociocultural: una perspectiva necesaria. Salud Pública de México. 2013;55:512-518. doi: 10.21149/spm.v55i5.7252.

21. Menéndez E. Epidemiología sociocultural: propuestas y posibilidades. Región y Sociedad. 2008;XX(2):5-50.

22. Kiefer C. Doing health anthropology: Research methods for community assessment and change. New York: Springer Publishing Company; 2007.

23. Fassin D. Entre las políticas de lo viviente y las políticas de la vida. Hacia una antropología de la salud. Revista Colombiana de Antropología. 2004;40:283-318.

24. Singer M. The application of theory in medical anthropology: an introduction. Medical Anthropology. 1992; 14(1):1-8.

25. Breilh J. Epidemiología crítica: Ciencia emancipadora e interculturalidad. Buenos Aires: Lugar Editorial; 2003.

26. Douglas M, Wildavsky A. Risk and culture: An essay on the selection of technological and environmental dangers. Berkeley: University of California Press; 1982.

27. Mitchell T. Colonial trauma and political pathways to healing [Internet]. En: Stewart SL, Moodley R, Hyatt A, (eds.). Indigneous cultures and mental health counselling: Four directions for integration with counselling psychology [Internet]. New York: Routledge; 2017 [citado 1 jun 2018]. p. 141-154. Disponible en: https://tinyurl.com/ уу5 vpajm.

28. Duran E, Duran B, Heart MYHB, Horse-Davis SY. Healing the American Indian soul wound. En: Danieli Y, (ed.). International handbook of multigenerational legacies of trauma. Boston: Springer; 1998. p. 341-354. doi: 10.1007/978-1-4757-55671_22. 
29. Evans-Campbell T. Historical trauma in American Indian/Native Alaska communities: a multilevel framework for exploring impacts on individuals, families, and communities. Journal of Interpersonal Violence. 2008;23(3):316-338. doi: 10.1177/0886260507312290.

30. Kirmayer LJ, Brass GM, Tait CL. The mental health of aboriginal peoples: transformations of identity and community. Canadian Journal of Psychiatry. 2000;45(7):607-616. doi: 10.1177/0706 74370004500702 .

31. Harwood JT, Giles H, Bourhis R. The genesis of vitality theory: Historical patterns and discoursal dimensions. International Journal of the Sociology of Language. 1994;108(1):167-206. doi: 10.1515/ ijsl.1994.108.167.

32. Guitart ME, Rivas Damián MJ, Pérez Daniel MR. Identidad étnica y autoestima en jóvenes indígenas y mestizos de San Cristóbal de las Casas (Chiapas, México). Acta Colombiana de Psicología. 2011;14(1):99-108.

33. Román M, Moreno M. Autoestima en jóvenes indígenas: Borucas y Terrabas. Revista de Ciencias Sociales. 2013. doi: 10.15517/rcs.v0i126127.8786

34. Abbott P, Chase DM. Culture and substance abuse: Impact of culture affects approach to treatment. Psychiatric Times. 2008;25(1):43-46.

35. Vasilachis de Gialdino I, (coord.). Estrategias de investigación cualitativa. Barcelona: Gedisa; 2006.

36. Ferrarotti F. Sobre a autonomia do método biográfico. En: Nóvoa A, Finger M, (orgs.). O método (auto) biográfico e a formação. Lisboa: Ministério da Saúde, Centro de Formação e Aperfeiçoamento Profissional; 1988. p. 17-34.

37. Glaser B, Strauss A. The discovery of grounded theory: Strategies for qualitative research. Chicago: Aldine Publishing Company; 2006.

38. Martínez-Hernáe A, Correa-Urquiza M. Un saber menos dado: nuevos posicionamientos en el campo de la salud mental colectiva. Salud Colectiva. 2017;13(2):267-278. doi: 10.18294/ sc. 2017.1168 .

39. Salas R. Filosofía mapuche. En: Dussel E, Mendieta E, Bohórquez C, (eds.). El pensamiento filosófico latinoamericano, del Caribe y "Latino" (1300-2000). Ciudad de México: Siglo XXI Editores, Centro de Cooperación Regional para la Educación de Adultos en América Latina y el Caribe; 2009. p. 41-46.
40. Saaresranta T. Perspectivas hacia una educación intracultural en el contexto indígena originario campesino. Tinkazos. 2011;14(30):127-144.

41. Aparicio Gervás JM, Delgado Burgos MA. Multiculturalidad, interculturalidad e intraculturalidad: tres conceptos de intervención social que coexisten en la sociedad latinoamericana del nuevo milenio. Entre 2 Orillas. 2011;9:11-15.

42. Zambrano A, Donoso E, Aguilera S, Candia A, Alarcón P. Influencia de la identidad cultural mapuche/lafkenche en la regulación del consumo de alcohol: la perspectiva de autoridades tradicionales mapuche/lafkenche del sector costero de la IX región de la Araucanía. Revista Cambios y Permanencias. 2018;9(1):324-356.

43. Hallett D, Chandler M, Lalonde C. Aboriginal language knowledge and youth suicide. Cognitive Development. 2007;22:392-399. doi: 10.1016/j. cogdev.2007.02.001.

44. Nichols F. Take the best from both cultures: An aboriginal model for substance use prevention and intervention. Aboriginal and Islander Health Worker Journal. 2010;34(3):10-14.

45. Komro KA, Livingston MD, Wagenaar AC, Kominsky TK, Pettigrew DW, Garrett BA, Cherokee Nation Prevention Trial Team. Multilevel prevention trial of alcohol use among American Indian and white high school students in the Cherokee Nation. American Journal of Public Health. 2017;107(3):453-459. doi: 10.2105/ AJPH.2016.303603.

46. Santos BS. Descolonizar el saber, reinventar el poder. Santiago de Chile: LOM ediciones; 2013.

47. Resnicow K, Soler R, Braithwaite RL, Ahluwalia JS, Butler J. Cultural sensitivity in substance use prevention. Journal of Community Psychology. 2000;28(3):271-290.

48. Okamoto SK, Helm S, Pel S, McClain LL, Hill AP, Hayashida JK. Developing empirically based, culturally grounded drug prevention interventions for indigenous youth populations. The Journal of Behavioral Health Services \& Research. 2014;41(1):8-19.

49. Dickerson D, Baldwin JA, Belcourt A, Belone L, Gittelsohn J, Kaholokula JK, Wallerstein N. Encompassing cultural contexts within scientific research methodologies in the development of health promotion interventions. Prevention science: the official journal of the Society for Prevention Research. 2018. doi: 10.1007/s11121018-0926-1. 
50. Zambrano A. Las diversas caras del poder: Poder para el desarrollo Humano. Revista Ecos. 2012;2(2):200-214.

51. Zambrano Constanzo A. Criterios de intervención en estrategias de empoderamiento comunitario: La perspectiva de profesionales y expertos de la intervención comunitaria en Chile y España. [Tesis de doctorado]. Barcelona: Universidad de Barcelona; 2007.

52. Lapalma A. El escenario de la intervención comunitaria. Revista de Psicología de la Universidad de Chile. 2001;X(2):61-70.
53. Montero M. Teoría y práctica de la psicología comunitaria: La tensión entre comunidad y sociedad. Barcelona: Paidós; 2005.

54. Bustamente G. Psicología comunitaria y pueblos indígenas ¿es posible una psicología comunitaria no colonialista? En: Zambrano A, Rozas G, Magaña I, Asun D, Pérez-Luco R, (coords.). Psicología comunitaria en Chile: Evolución, perspectivas y proyecciones. Santiago de Chile: Ril Editores; 2007. p. 293-323.

\section{FORMA DE CITAR}

Garcés Pérez GO, Zambrano Constanzo AX. Significados en torno al desarrollo del consumo problemático y dependencia alcohólica en comunidades mapuches rurales de la región de la Araucanía, Chile, 2016-2017. Salud Colectiva. 2019;15:e1932. doi: 10.18294/sc.2019.1932.

Recibido: 13 de junio de 2018 | Versión final: 11 de abril de 2019 | Aprobado: 24 de abril de 2019

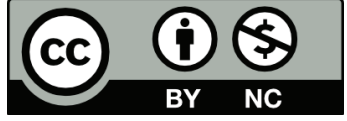

Esta obra está bajo una licencia de Creative Commons Reconocimiento-NoComercial 4.0 Internacional. Reconocimiento - Permite copiar, distribuir y comunicar públicamente la obra. A cambio, se debe reconocer y citar al autor original. No Comercial - Esta obra no puede ser utilizada con finalidades comerciales, a menos que se obtenga el permiso. 